\title{
Kriminalpolitische Anmerkungen zum Thema Jugendhilfe und Justiz ${ }^{1}$
}

Werner Nickolai

\begin{abstract}
ugend und Gewalt, Jugend und Kriminalität sind Themen, die uns seit Jahren beschäftigen. Die Jugendlichen werden scheinbar immer problematischer, Gewalthandlungen nehmen zu und die Kriminalität ist insbesondere Anfang der 90er Jahre gestiegen. So jedenfalls die veröffentlichte Meinung.
\end{abstract}

Die Gesellschaft ist zutiefst verunsichert. So wundert es nicht, dass immer härtere Maßnahmen gefordert werden; zumindest die geschlossene Unterbringung, wenn nicht gleich die Inhaftierung. Und dies hat Folgen: Im Bereich der Justiz nimmt nicht nur die Zahl der der jugendlichen Untersuchungshäftlinge zu. Auch im Jugendstrafvollzug ist ein stetiger Anstieg zu verzeichnen.

Ziel der Fachtagung war es, kritisch zu prüfen, ob aus dem Blick der Jugendhilfe eine Zusammenarbeit mit der Justiz möglich ist und wo die Grenzen der Zusammenarbeit verlaufen.

Als Vorsitzender der Katholischen Bundes-Arbeitsgemeinschaft Straffälligenhilfe (KAGS) im Deutschen Caritasverband ${ }^{2}$ und Professor für Soziale Arbeit mit Schwerpunkt Straffälligenhilfe an der Katholischen Fachhochschule Freiburg war es meine Aufgabe zu dem Spannungsverhältnis »Jugendhilfe und Justiz«Stellung zu beziehen.

Die kriminalpolitischen Leitlinien der KAGS lassen sich wie folgt umschreiben:

- Versöhnung statt strafen

- Integration statt Ausgrenzung

Mit Blick auf unsere kriminalpolitische Maxime der »Integration statt Ausgrenzung « hat die KAGS entschieden Position gegen die »Lebenslange Freiheitsstrafe «, wie auch gegen die "Sicherungsverwahrung « bezogen. Die Arbeitsgemeinschaft hat sich auch öffentlich gegen die Verschärfung des Jugendstrafrechts gewandt und sich anlässlich einer von ihr ver- anstalten Tagung 1995 zum Thema »Justiz oder Jugendhilfe?« gegen die (Wieder-) Einführung geschlossener Einrichtungen in der Jugendhilfe ausgesprochen. Die letzt genannte Positionierung muss allerdings neu überdacht werden.

Eine Positionierung zur Frage der Abschaffung des derzeit bestehenden Jugendstrafvollzugs, wie ihn Heinz Cornel schon 1984 fordert, der Untertitel seines Buches »Geschichte des Jugendstrafvollzugs « lautet »Ein Plädoyer für seine Abschaffung « hat die KAGS noch nicht vorgenommen, so dass die weiteren Ausführungen meine persönliche Einstellung und nicht die der KAGS wiedergibt.

Dass der Jugendstrafvollzug sein in § 91 Absatz 1 Jugendgerichtsgesetz formuliertes Ziel »den Verurteilten dazu zu erziehen, künftig einen rechtschaffenen und verantwortungsbewussten Lebenswandel zu führen", nur schwer und überwiegend gar nicht erreicht, lässt sich nicht nur an den Rückfallzahlen festmachen.

So schreibt etwa Horst Viehmann $(1995,21)$, ehemals Referent für Jugendstrafrecht im Bundesjustizministerium, dass »nahezu einhellig unter Experten die Erziehungsfähigkeit vollstreckter Erziehungs- und Jugendstrafe bezweifelt und die Zweifel größer werden, je länger die Verbüßungszeit ist «. Und er fährt fort: »Die unterstellten positiven Wirkungen der Kriminalitätsverhinderung stellen sich eben nicht ein. Für künftiges rechtsfreies Verhalten bestrafter junger Menschen haben vollstreckte Freiheitsstrafen eher gegenteilige Effekte, wirken eher Kriminalität verfestigend ... Die Rückfallgefahr nach der Entlassung wird größer! Die Gefährlichkeit des Täters nimmt zu! Je länger, desto entsozialisierter .

Joachim Walter (1993), Anstaltsleiter in der Jugendvollzugsanstalt Adelsheim, stellt fest, dass der Jugendstrafvollzug keine pädagogische Institution im eigentlichen Sinne des Wortes ist. Er verweist auf die von Goff- mann (1972) beschriebene «totale Institution « mit ihrem gestaffelten Autoritätssystem, das die Insassen nahezu umfassend bevormundet und vereinnahmt. Die fundamentale Trennung zwischen Insassen und Aufsichtspersonal geht im Prinzip soweit, dass es jedem Bediensteten gestattet ist, jeden Gefangenen zu disziplinieren. Auf die Aufzählung weiterer Merkmale der »totalen Institution", die alle letztlich darauf abzielen, die Identität des Menschen zu beschädigen, sei an dieser Stelle verzichtet. Verhaltensregeln werden in der »totalen Institution" grundsätzlich nicht pädagogisch gesehen und bewertet. Walter kommt deshalb zu dem Schluss, dass der Jugendstrafvollzug eher ein Disziplinierungsinstrument als eine pädagogische Institution darstellt.

Für die Erziehungswissenschaft hat die Strafe in der Erziehung nur dort ihren Platz, wo sie erstens in die Beziehung von Erwachsenen und Kindern integriert ist, wo sie zweitens die Grundlage dieser Beziehung nicht zerstört und wo sie drittens die Möglichkeit der Auseinandersetzung mit der Verfehlung nicht blockiert (Müller 1993, 221). Diese Kriterien treffen auf die Jugendstrafe gar nicht und auf die Disziplinarmaßnahmen innerhalb des Vollzug kaum zu.

Aber auch die Erziehungswissenschaft steht der Strafe eher ablehnend gegenüber, denn Strafe erzeugt eher Angst vor der Bestrafung und steuert damit das Verhalten-wenn überhaupt-auf der untersten Stufe der Moralität.

Schauen wir uns die Ergebnisse der Sanktionsforschung an, wie sie uns Jehle, Heinz und Sutterer 2003 in einer umfassenden Studie für alle Sanktionsformen vorgelegt haben, so ist $\mathrm{zu}$ konstatieren, dass der Jugendstrafvollzug die höchste Rückfallquote aller Sanktionsformen nach dem Jugendgerichtsgesetz vorweist. So werden nach einer verbüßten Jugendstrafe im Laufe von vier Jahren $78 \%$ erneut rückfällig und $45 \%$ keh- ren gar wieder in den Strafvollzug zurück.

Die erziehungsfeindlichen Grundbedingungen der totalen Institution Strafvollzug, seine subkulturelle Problematik wie auch die Drogenproblematik werden vom Justizministerium Baden-Württemberg gesehen. Dies war mit ausschlaggebend, dass das Ministerium mit dem Projekt Chance neue Wege im Jugendstrafvollzug gehen will. Für Rüdiger Wulf, Referent im Justizministerium BadenWürttemberg, ist der jetzige Jugendstrafvollzug kein guter Ort für Jugendliche.

Langfristig, so Wulf in einem Referat bei einer Tagung in der Evangelischen Akademie Arnoldshain, soll es auf eine Dreigliederung des Strafvollzugs hinaus laufen. Im Jugendstrafvollzug sollen sich nur oder überwiegend Jugendliche befinden. Der Jungtätervollzug wäre dann für die Altersgruppe der 18- bis 24- oder 27jährigen zuständig und der Erwachsenenvollzug für die älteren Gefangenen.

Eine weitere Ausdifferenzierung stellt nun der Jugendstrafvollzug in freien Formen dar, dessen Rechtsgrundlage im $\S 91$ Absatz 3 zu finden ist. Die Jugendstrafe wird, wie wir am Beispiel des Projekts Chance gesehen haben, in einer Jugendhilfeeinrichtung vollstreckt. Angesprochen sind hier Jugendliche und nur in Ausnahmefälle ältere Probanden bis 21 Jahren. Der Ausbau solcher Einrichtungen ist beschlossene Sache.

Bevor ich zu einer weiteren Einschätzung des Jugendstrafvollzugs in freier Form komme, möchte ich kurz weitere kriminalpolitische Forderungen zur Bekämpfung der Jugendkriminalität benennen, wie wir sie nicht nur aus Baden-Württemberg kennen.

Der Pressemitteilung des Justizministeriums Baden-Württemberg vom 10. August 2005 ist zu entnehmen, dass Justizminister Goll für die 18 bis 21-jährigen Straftäter die Anwendung des Erwachsenenstrafrechts fordert. »Wenn aber 18 bis 21-jährige Straftäter schon nicht nach Erwachsenenstrafrecht verurteilt werden «, so Goll, »wäre eine Erhöhung des Strafrahmens von 10 auf 15 Jahre der richtige Schritt«. Daneben macht sich Goll für die Einführung eines Warnschussarrests stark. Der Warnschussarrest 
käme bei einer Verurteilung zu einer Jugendstrafe, die zur Bewährung ausgesetzt wird, zum Zuge.

Neben diesen Forderungen ist immer wieder auch zu hören, dass die Strafmündigkeit auf 12 Jahre herabgesetzt werden soll. Dem Wahlprogramm der Bundes-CDU, ist zu entnehmen, dass bei den Heranwachsenden die Sicherungsverwahrung anwendbar sein soll. Hier plädiert sie auch für die Herausnahme der Heranwachsenden aus der Jugendgerichtsbarkeit. Für das Jugendstrafrecht fordert sie die Einführung der nachträglichen Sicherungsverwahrung.

Einerseits wird der Versuch unternommen, Jugendstrafvollzug in freier Form durchzuführen. Andererseits werden Forderungen nach längerer Jugendstrafe bis hin zur Sicherungsverwahrung laut. Wie passt das zusammen?

Schauen wir zunächst noch auf die Strafunmündigen, also auf die unter 14jährigen. Anlässlich einer Veranstaltung in der Universität Potsdam hat die brandenburgische Justizministerin Beate Blechinger ihre Vorstellung zur Bekämpfung der Jugendkriminalität vorgestellt: Für sie ist ein erheblicher Teil der Eltern regelrecht erziehungsunfähig. Um diese Lücke zu schließen seien Kooperationsmodelle erforderlich, die Familien, Kindergärten, Schulen und Jugendämter frühzeitiger vernetzen. $\mathrm{Zu}$ überlegen sei auch, ob nicht eine Rückkehr zur früheren Fürsorgeregelung nötig sei, um zu verhindern, dass die staatliche Hilfe zur Erziehung durch die Verweigerung der Erziehungsberechtigten blockiert werden können. Der wiederholt schwerwiegende Verstoß von Kindern gegen Strafgesetze sei ein Indikator für die Gefährdung des Kindeswohles, so dass es sinnvoller sei, die »Problemkinder « in Einrichtungen der Jugendhilfe unterzubringen anstatt für alle die Strafmündigkeit zu senken.

Wenn auch die Diagnose von Beate Blechinger, die das Versagen der Familien in den Mittelpunkt ihrer Überlegungen stellt, für mein Dafürhalten zu kurz greift, so stimme ich ihr doch zu, wenn sie sich gegen die Herabsenkung der Strafmündigkeit ausspricht, weil sie glaubt, dass die Jugendhilfe gegenüber der Justiz hier die besseren Angebote machen kann.
Genau dies sehen wir ja auch bei der Vollstreckung der Jugendstrafe in freier Form, also in einer Jugendhilfeeinrichtung. In einer jüngst vorgelegten Studie über das Projekt Chance kommt der Autor Christian Biendl (2005, 137f.) zu folgendem positiven Ergebnis: »Die Umsetzung durch das Projekt Chance ist sehr gelungen. Es werden Haftschäden vermieden und eine positive Atmosphäre geschaffen, in der konstruktiv an Veränderungen gearbeitet werden kann. Dadurch werden gute Voraussetzungen für eine spätere Legalbewährung geschaffen«.

Der Jugendstrafvollzug hat über Jahrzehnte hinweg mehr oder weniger, eher mehr als weniger, versagt. Es gibt für mich keinen Grund an ihm festzuhalten. Mag sein, dass man die Abschaffung des Jugendstrafvollzugs nicht fordern kann, ohne sich zu überlegen, wie Schritte dahin aussehen können. Ich würde mir wünschen, dass das Modell »Jugendstrafvollzug in freier Form « ein Modell ist, das eine Perspektive eröffnet, langfristig den konkret existierenden Jugendstrafvollzug überflüssig $\mathrm{zu}$ machen bzw. ihn so unter Legitimationsdruck zu setzen, dass er sich ändern bzw. öffnen oder ganz verschwinden muss.

Ein Blick in die Schweiz zeigt uns, dass die stationäre Jugendhilfe auch Jugendlichen Angebote machen kann, die straffällig geworden sind. Warum kann die Schweiz auf einen Jugendstrafvollzug, wie wir ihn kennen, verzichten und wir nicht?

Abschließend darf ich meine kriminalpolitischen Vorstellungen doch noch kundtun:

- Ich schließe mich der Forderung der Deutschen Vereinigung für Jugendgerichte und Jugendgerichtshilfen e.V. an, die sich für eine Heraufsetzung der Strafmündigkeit auf 16 Jahren ausgesprochen hat.

- Die Herausnahme der Heranwachsenden aus dem Jugendgerichtsgesetz ist mir nicht plausibel.

- Der Jugendarrest als Zuchtmittel ist ersatzlos zu streichen. Einen Warnschussarrest darf es nicht geben.

- Jugendstrafe kann nicht wegen »schädlichen Neigungen« ausgesprochen werden. Dies darf nur bei Schwere der Schuld erfolgen.

- Die Jugendstrafe darf fünf Jahre nicht übersteigen.
- Die Jugendstrafe darf nur in einer Jugendhilfeeinrichtung vollstreckt werden.

- Sicherungsverwahrung für Heranwachsende und die nachträgliche Sicherungsverwahrung für Jugendliche sind abzulehnen.

- Die Untersuchungshaftvermeidung nach $\S \S 71,72$ JGG ist weiter auszubauen und auf die Heranwachsenden auszudehnen.

- Das derzeit bestehende System von Jugendhilfe und Jugendstrafrechtspflege muss sicherlich überdacht werden. Jugendhilfeeinrichtungen sind besser als der Jugendstrafvollzug in der Lage auch straffällig gewordenen Menschen gerecht $\mathrm{zu}$ werden. Im Rahmen der Erziehungsmaßregeln, sei es als Weisung nach $\S 10$ JGG, als Hilfe zur Erziehung nach § 12 JGG oder als Bewährungsauflage, haben sie mit diesem Klientel schon zu tun.

\section{Literatur}

Biendl, Christian: Jugendstrafvollzug in freier Form - am Beispiel des »Projekt Chance «. Konstanz 2005.

Cornel, Heinz: Geschichte des Strafvollzugs. Ein Plädoyer für seine Abschaffung. Weinheim Basel 1984.

Goffman, Erving: Asyle. Über die soziale Situation psychiatrischer Patienten und anderer Insassen. Frankfurt 1972.

Kohlberg, Lawrence / Scharf, Peter / Hickey, Joseph: Die Gerechtigkeitsstruktur im Gefängnis - Eine Theorie und eine Intervention. In: Portele,G.: Sozialisation und Moral. Weinheim 1978.

Müller, Siegfried: Erziehen - Helfen -Strafen. Zur Klärung des Erziehungsbegriffs im Jugendstrafrecht aus pädagogischer Sicht. In: Peters,H.(Hrsg.): Muß Strafe sein? Oldenburg 1993, S.217-233.

Jehle, Jörg-Martin / Heinz, Wolfgang/Sutterer, Peter: Legalbewährung nach strafrechtlichen Sanktionen. Eine kommentierte Rückfallstatistik. Berlin 2003.

Pressemitteilung des brandenburgischen Ministerium der Justiz vom 27. Juni 2005.

Pressemitteilung des baden-württembergischen Justizministerium vom 10 . August 2005.

Viehmann, Horst: Verschärfung des Strafrechts - Eine geeignete Antwort auf neue Dimensionen der Jugendkriminalität? In: Reindl, Richard / Kawamura, Gabriele / Nickolai, Werner (Hrsg.): Prävention - Entkriminalisierung - Sozialarbeit. Alternativen zur Strafverschärfung. Freiburg 1995.

Walter, Joachim: Jugendstrafvollzug auf dem Weg zu einer pädagogischen Institution? In: Elbing / Gehl / Nickolai / Reindl(Hrsg.): Jugendstrafvollzug zwischen Erziehen und Strafe. Pädagogische Ansätze - Konzepte - Perspektiven. Saarbrücken 1993, S.104-120.
Wulf, Rüdiger: Umgang mit jungen Mehrfach- und Intensivtätern im Jugendstrafrecht und Jugendstrafvollzug. Vortrag am 21. März 2004 in der Evangelischen Akademie Arnoldshain. www.evangelischeakademie.de/materialien/045758/ Wulf.pdf.

Werner Nickolai ist Professor an der Katholischen Fachhochschule in Freiburg und Vorsitzender der Katholischen Bundes-Arbeitsgemeinschaft Straffälligenhilfe.

\section{Anmerkungen}

1 Fachtagung »Jugendhilfe und Justiz am 4. - 6.10.2005 in Frankfurt. Veranstalter: Referat Jugendhilfe des Deutschen Caritasverbandes e. V. Freiburg in Kooperation mit: Referat Basisdienste und besondere Lebenslagen des Deutschen Caritasverbandes, Bundesverband katholischer Einrichtungen und Dienste der Erziehungshilfe e.V., Katholische Bundes-Arbeitsgemeinschaft Straffälligenhilfe.

2 Der Katholische Bundes-Arbeitsgemeinschaft Straffälligenhilfe, 1927 als »Katholische Reichsarbeitsgemeinschaft für Gerichtshilfe, Gefangenen- und Entlassenenfürsorge " gegründet, gehören an: der Deutsche Caritasverband, der Sozialdienst Katholischer Frauen, der SKM Katholischer Verband für soziale Dienste in Deutschland e.V., die Bundeskonferenz der Katholischen Seelsorge bei den Justizvollzugsanstalten in Deutschland und die Katholischen Träger der Straffälligenhilfe vor Ort. 